\title{
Potencial teórico, social y metodológico en los trabajos finales de graduación de la maestría de Enfermería Ginecológica, Obstétrica y Perinatal ${ }^{1}$
}

Institución: Universidad de Costa Rica

Ligia Rojas Valenciano ${ }^{2}$

Laura López Quirós ${ }^{3}$

Ana Ibis Campos González ${ }^{4}$

\section{COMO CITAR}

Rojas, L., López, L. y Campos, A. (2015). Potencial teórico, social y metodológico en los trabajos finales de graduación de la maestría de Enfermería Ginecológica, Obstétrica y Perinatal. Rev. Enfermería Actual en Costa Rica, 28, 1-19.

DOI:httn://dx.doi.org/10.15517/revenf.v0i28.17281

\section{RESUMEN}

Introducción. El objetivo de este artículo es presentar los resultados del proyecto de investigación $\mathrm{N}^{\mathrm{o}}$. 421-B2-A19 en cuanto al potencial teórico, social y metodológico en los trabajos finales de graduación realizados en la maestría de Enfermería Ginecológica, Obstétrica y Perinatal. El componente de investigación en un área del quehacer de la Enfermería, cuya meta es preparar a los profesionales en enfermería clínica acerca de utilizar diligentemente la investigación.

Método. Se aplicó la investigación documental como una variante de la investigación científica cuyo objetivo fundamental es el análisis de diferentes fenómenos utilizando técnicas muy precisas de la documentación existente que, directa o indirectamente, aporte la información. Se incluyó 37 trabajos de investigación aplicada, 22 sustentados en el enfoque cualitativo y 15 en el cuantitativo, desde el año 2003 al 2011. A cada trabajo se le aplicó un análisis crítico utilizando una matriz construida a partir de elementos recomendados por CASpe para valorar documentos científicos cuantitativos (Critical Appraisal Skills Programme Español). Para el análisis crítico de los trabajos finales de investigación de corte cualitativo también se construyó una matriz que incluye aspectos del CAT (Critically Appraised Topic): EBCP (Evidence-Based Clinical Practice).

Resultado. Los trabajos finales de graduación no demostraron ser significativos en el cambio o mejoramiento de la práctica profesional en términos de mejorar la calidad de atención a las personas. Solo han sido relevantes para que se gradúe un grupo grande de estudiantes. Existe poca claridad -en general- de los aspectos metodológicos de los enfoques investigativos cualitativo y cuantitativo.

Conclusión. La investigación en la maestría Ginecológica, Obstétrica y Perinatal no aporta un conocimiento enfermero sustantivo, lo cual provoca poco desarrollo disciplinar. Los trabajos finales de graduación aparentan ser solo requisito de graduación, por tanto, no contribuyen al mejoramiento de las prácticas ginecológicas, obstétricas y perinatales.

Palabras clave: enfermería, investigación, obstetricia, posgrado.

\footnotetext{
${ }^{1}$ Fecha de recepción: 2 de octubre del 2014

Fecha de aceptación: 15 de noviembre del 2014

${ }^{2}$ Enfermera. Escuela de Enfermería. Universidad de Costa Rica. Costa Rica. Correo electrónico: ligiarojas7@gmail.com

${ }^{3}$ Enfermera. Escuela de Enfermería. Universidad de Costa Rica. Costa Rica. Correo electrónico: 1auralopez07@gmail.com

${ }^{4}$ Enfermera. Escuela de Enfermería. Universidad de Costa Rica. Costa Rica. Correo electrónico: ibiscampos@gmail.com
} 


\title{
Theoretical, methodological and social potential in the final works of the Master Degree in Gynecological, Obstetric and Perinatal Nursing ${ }^{1}$
}

Ligia Rojas Valenciano ${ }^{2}$ Laura López Quirós ${ }^{3}$

Institution: University of Costa Rica

Ana Ibis Campos González ${ }^{4}$

\section{CITED:}

Rojas, L., López, L. y Campos, A. (2015).Theoretical, methodological and social potential in the final works of the master degree in Gynecological, Obstetric and Perinatal Nursing. Rev. Enfermería Actual en Costa Rica, 28, 1-19.

DOI:http://dx.doi.org/10.15517/revenf.v0i28.17281

\begin{abstract}
Introduction. The aim of this paper is to present the results of the research project $\mathrm{N}^{\mathrm{o}}$. 421-B2-A19 compared to the theoretical, social and methodological potential in the final works of the Masters Degree in Gynecological, Obstetric and Perinatal Nursing. The research component in an area of nursing, whose goal is to prepare professional clinical nurses about using research diligently.

Method. Documentary research was applied as a variant of scientific research whose main objective is the analysis of different phenomena using very precise techniques of existing documentation, which directly or indirectly provide the information. 37 applied research projects, 22 in the qualitative approach and 15 in quantitative approach, from 2003 thru 2011. Each job was applied by using a critical analysis built from CASpe recomendations. We constructed a matrix for assessing documents of the quantitative approach (Critical Appraisal Skills Programme Spanish). For critical analysis of the final work of qualitative research, a matrix was also constructed using aspects of CAT (Critically Appraised Topic): EBCP (Evidence-Based Clinical Practice).

Result. The final graduation work proved not prevailing in change or improvement of professional practice in terms of improving the quality of care given to people. They have only been relevant for a large group of postgraduate students. There is little clarity, in general, of the methodological aspects of both research approaches: qualitative and quantitative.

Conclusion. Research Master Degree in Gynecologic, Obstetric and Perinatal nursing does not provide a substantive nursing knowledge, which leads to poor disciplinary development Final papers appear to be only a graduation requirement, which does not contribute to the improvement of Gynecologic, Obstetric and Perinatal nursing practices based on research.
\end{abstract}

Keywords: nursing, obstetrics, master-degree, research.

\footnotetext{
${ }^{1}$ Date of receipt: october 2, 2014

Date of acceptance: november 15, 2014

${ }^{2}$ Nurse. School of Nursing. University of Costa Rica. Costa Rica. E-mail: ligiarojas7@gmail.com

${ }^{3}$ Nurse. School of Nursing. University of Costa Rica. Costa Rica. E-mail: lauralopez07@gmail.com

${ }^{4}$ Nurse. School of Nursing. University of Costa Rica. Costa Rica. E-mail: ibiscampos@gmail.com
} 


\section{INTRODUCCIÓN}

El Programa de Posgrado, junto con un plan de estudios que conduce en este momento a la obtención de la Maestría Académica en Ciencias de la Enfermería, representan factores que justificaron la apertura de la Maestría en Enfermería Ginecológica, Obstétrica y Perinatal. Fue entonces que la subcomisión de esa maestría elaboró una propuesta, aprobada en la modalidad profesional, como respuesta a las nuevas tendencias, condiciones de vida y perfil epidemiológico del país (CONARE, 2001).

Respecto de la Enfermería Ginecológica, Obstétrica y Perinatal, esta es

un área de acción centenaria en el país, que se ha caracterizado por una ampliación de su quehacer conforme han variado las necesidades de salud de la población, su perfil epidemiológico, los programas de salud, las políticas de salud y las nuevas tendencias en tecnologías en la atención ginecológica, obstétrica y perinatal a nivel mundial y nacional, en pro del mejoramiento de la calidad de vida de la mujer y del hombre en su ciclo de vida. Por lo tanto, "el reto actual es la búsqueda de abordajes para la transformación de los entornos de trabajo a nivel individual y colectivo con base en la equidad y respecto de las diferencias" (Consejo Nacional de Rectores Oficina de Planificación de la Educación Superior, 2001, p.2-3).

Dentro de la investigación, se destaca la actividad gestora del estudiantado que se enfoca en

la interacción de la persona y el entorno, para lo cual el cuidado se fundamenta en la construcción del conocimiento en Enfermería Ginecoobstétrica y Perinatal; ambos elementos se apoyan en la investigación principalmente, para la construcción de la atención integral de salud en las etapas preconcepcional, concepcional, parto, postnatal, y neonatal en la adolescencia, juventud y adultez, mediante estrategias de prevención, promoción, curación, rehabilitación, en escenarios y niveles de atención de la salud (Consejo Nacional de Rectores Oficina de Planificación de la Educación Superior, 2001,p.5).

Además, la práctica que realiza el estudiantado genera preguntas, hipótesis de investigación, se prueba teorías y nuevas propuestas, prácticas que, a su vez, mejorará la atención a las personas.

Respecto de lo anterior, cabe destacar que con la investigación,

el proceso educativo de estudiantes y docentes apuntará a describir, explicar y predecir los fenómenos por medio de diferentes tipos de investigación en el abordaje de los problemas de salud ginecológica, obstétrica y perinatal, en forma creativa, tolerante y de reconocimiento de la importancia de la evidencia clínica y social (Consejo Nacional de Rectores Oficina de Planificación de la Educación Superior, 2001,p.6).

La investigación se ha definido de muchas maneras: Fawcett y Garity (2009) la definen como un proceso 


\section{.}

riguroso, formal y sistemático usado para generar y evaluar teorías, mas no para solucionar problemas, debido a que su eje es el desarrollo del conocimiento para mejorar la comprensión, mientras que la resolución de problemas se centra en el uso de conocimientos existentes para resolver problemas prácticos (Fain, 2004). Más precisamente, el conocimiento desarrollado por medio de la investigación no ofrece respuestas a los problemas, sino que ayuda a pensar diferente acerca de los problemas que encontramos en la práctica (Fawcett y Garity, 2009).

Respecto de lo anterior, el componente de investigación en un área del quehacer de la Enfermería, en este caso, en la maestría Ginecológica, Obstétrica y Perinatal, tiene como meta preparar a los profesionales en enfermería clínica en cuanto a utilizarla diligentemente, sin obviar la evaluación, la identificación de problemas dentro de la práctica clínica, el conocimiento de los resultados de la práctica y las aplicaciones clínicas de la investigación (American Association of Colleges of Nursing, 1996, p.6).

Partiendo de lo anterior, la importancia de la investigación radica en diversos motivos: tal vez ayude a resolver un problema social o a construir una teoría, aunque no hay que olvidar que sin embargo, lo que es relevante para algunas personas no lo es para otras, razón por la que se han establecido algunos criterios flexibles y no exhaustivos para evaluar el potencial de un trabajo de investigación, tales como "la conveniencia, la relevancia social, el valor teórico y la utilidad metodológica" (Hernández, Fernández-Collado y Baptista, 2010, p.15).

En ese sentido, en este trabajo de investigación se pretende analizar las temáticas que han desarrollado los estudiantes en los trabajos finales de investigación realizados en la maestría de Enfermería Ginecológica, Obstétrica y Perinatal de la Universidad de Costa Rica y determinar en cada uno de ellos el potencial teórico, social o metodológico, como lo establece el autor mencionado, máxime considerando que Enfermería siempre ha utilizado los hallazgos como apoyo de la recolección de datos, intervenciones y evaluación en el cuidado de las personas, a lo que ha llamado "utilización de la investigación".

$\mathrm{Al}$ investigar en una maestría, conviene monitorizar dichos procesos, de modo que se esclarezca las líneas de investigación, las preferencias del estudiantado en relación con investigar ciertos temas específicos de acuerdo con las necesidades detectadas en las diferentes prácticas clínicas. Tal proceso fortalece la práctica en Enfermería Ginecológica, Obstétrica y Perinatal, siempre y cuando los resultados de las investigaciones sean considerados como pruebas, dado que son evidencia necesaria para la práctica. Por eso no es sorprendente considerar que la práctica y la investigación son esencialmente el mismo proceso: es decir, el de enfermería y el de investigación en enfermería envuelven el mismo pensamiento crítico de habilidades y acciones en las que confluyen la rigurosidad y sistematización tanto de la práctica profesional como de la investigación, dado que de la práctica surgen ideas para investigar y la investigación mejora la práctica, durante la que se identifica un problema para el se desarrolla un plan que luego será implementado y evaluado.

El impacto que la investigación tiene en la toma de decisiones significancia y, en el caso de Enfermería, ha demostrado que a partir de sus investigaciones se han elaborado políticas de salud y cambios en la legislación. Lo anterior se corrobora con una investigación pionera de Ann Rogers en Pensilvania (2004), la cual todavía se cita como referencia de cambios en la legislación estatal de los Estados Unidos en cuanto a restringir el uso de las 
horas extraordinarias obligatorias para las enfermeras. En su estudio se detectó que el riesgo de error se incrementa tres veces cuando las enfermeras trabajan 12,5 horas consecutivas o más; la relevancia de este trabajo estriba en que fue el primero en establecer un vínculo claro entre las horas trabajadas por enfermeras y un mayor riesgo de errores en el trabajo, además de que su impacto trasciende los cambios de la política en los EE.UU.

El vincular la investigación con los debates actuales se debe a la necesidad de enmarcar los resultados de investigación en el contexto del actual debate y las decisiones políticas (Barnsteiner, 2011), máxime considerando que los profesionales en enfermería poseen un conocimiento fundamental para guiar a los responsables políticos sobre la forma de mejorar la prestación de la atención; incluso, es una obligación social.

Lo expuesto, evidencia claramente la necesidad de llevar a cabo un análisis crítico de los trabajos de investigación con el fin de orientar el futuro desarrollo de la investigación en la maestría, de que se aplique la investigación en el quehacer práctico de la Enfermería y de que se constate que la práctica conduce a llevar a cabo investigación para desarrollar conocimientos, los cuales sirven para comprender y resolver problemas prácticos que, en este caso, atañen a la Enfermería Ginecológica, Obstétrica y Perinatal.

Como síntesis, se afirma que la Enfermería Ginecológica, Obstétrica y Perinatal tiene una connotación multidimensional que incluye lo físico-biológico, psicológico, social, cultural, y de valores, lo cual lleva a establecer áreas de práctica (atención) a partir de la investigación y esta a partir de la experiencia práctica, procesos que durante la formación del estudiantado culminan en un trabajo final de investigación aplicada.

Es importante destacar que aunque este no es un estudio nuevo, sí es novedoso en la maestría, puesto que hasta el momento no se conoce ninguna investigación formal que trate de responder a los objetivos planteados.

El objetivo fue analizar el potencial teórico, social y metodológico en los trabajos finales de graduación de la maestría de Enfermería Ginecológica, Obstétrica y Perinatal.

\section{MATERIALES Y MÉTODO}

Este trabajo se tipifica como una investigación documental, una variante de la investigación científica, cuyo objetivo fundamental es el análisis de diferentes fenómenos, para lo cual se emplea técnicas muy precisas para tratar toda la documentación existente que, directa o indirectamente, aporte información. Se observó y reflexionó sistemáticamente sobre realidades, tarea en la que se utilizó diferentes clases de documentos. En el caso de esta investigación, se utilizó trabajos finales de investigación aplicada realizados por el estudiantado en la maestría en Enfermería Ginecológica, Obstétrica y Perinatal, cuyo periodo de búsqueda abarca del año 2003 al 2011. En el estudio, se incluyó 37 trabajos de investigación aplicada, 22 sustentados en un enfoque cualitativo y 15 , en el cuantitativo.

Entre las características de este tipo de investigación, se destaca la recolección de documentos, el uso de procedimientos lógicos y mentales (análisis, síntesis, deducción, inducción entre otros). Al respecto, es necesario enfatizar que con una recopilación adecuada de datos se redescubre hechos, se sugiere problemas y busca otras fuentes de investigación, entre otros aspectos. 
El trabajo se desarrolló de forma ordenada y con objetivos precisos, los cuales son la base para la construcción de conocimientos. Se utilizó diferentes técnicas de localización y fijación de datos, análisis de documentos y de contenidos.

1. Para el acopio de información, se detectó todos los trabajos finales de investigación elaborados durante el periodo 2003- 2011.

2.Posteriormente, se elaboró fichas bibliográficas con los datos básicos del documento (nombre del trabajo final de graduación, autor (es), lugar de realización del trabajo de campo, fecha, modalidad); con la información, se confeccionó el registro en las fichas, con el que se localizó rápidamente el material en el momento oportuno.

3. Luego, se efectuó una lectura rápida del material recopilado para ubicar las variables estudiadas: el tema del trabajo y su correspondencia con una línea de investigación de la maestría, la relevancia social, teórica y metodológica.

4. De cada documento, se leyó la introducción, la justificación, el marco teórico o referencial, la metodología y los resultados, así como conclusiones y recomendaciones.

5. Para procesar y analizar la información, a cada trabajo se le aplicó un análisis crítico utilizando una matriz construida a partir de elementos recomendados por CASpe para valorar documentos científicos cuantitativos (Critical Appraisal Skills Programme Español). Para el análisis crítico de los trabajos finales de investigación de corte cualitativo, también se construyó una matriz usando aspectos del CAT (Critically Appraised Topic): EBCP (Evidence-Based Clinical Practice).

6. La información fue colocada en las matrices para su posterior análisis de acuerdo con el potencial teórico, social y metodológico.

7. Finalmente, se elaboró tablas que presentan los resultados según los objetivos establecidos en este proyecto.

\section{Consideraciones éticas}

Se guardó rigurosamente la identidad de los autores, tutores y los títulos de los diferentes trabajos de investigación aplicada usados en el análisis.

\section{RESULTADOS}

A continuación, se presentan los resultados según el enfoque de investigación planteado en cada uno de los trabajos de investigación aplicada y las dimensiones y variables anotadas en las matrices elaboradas para la recolección de información. También se reseña la escogencia temática del estudiantado para realizar los trabajos finales de investigación aplicada de acuerdo con las líneas de investigación propuestas por la maestría. 


\section{Escogencia temática}

En la tabla 1 se muestra la escogencia temática de las investigaciones aplicadas que se analizó en este estudio.

\section{Tabla 1}

Maestría GOP-UCR: Distribución de las temáticas investigadas por el estudiantado según ejes y líneas de investigación de la maestría presentados durante los años 2003 al 2011, 2013.

(Frecuencias absolutas y relativas)

\begin{tabular}{l|c|c|c|c|c|c|c|c}
\hline \multirow{2}{*}{\multicolumn{1}{c|}{ Ejes/línea }} & \multicolumn{2}{c|}{ Gerencia } & \multicolumn{2}{c|}{ Educativo } & \multicolumn{2}{c|}{ Clínico } & \multicolumn{2}{c}{ Total } \\
\cline { 2 - 10 } & $f i$ & $h i$ & $f i$ & $h i$ & $f i$ & $h i$ & $f i$ & $h i$ \\
\hline $\begin{array}{l}\text { Salud Sexual y Salud Reproductiva, } \\
\text { Derechos sexuales y reproductivos }\end{array}$ & 4 & 10,8 & 2 & 5,4 & 4 & 10,8 & 10 & 27,02 \\
\hline Enfermería Obstétrica & 12 & 32,43 & 2 & 5,4 & 8 & 21,62 & 22 & 59,45 \\
\hline $\begin{array}{l}\text { Enfermería Ginecológica y } \\
\text { andrológica }\end{array}$ & 1 & 2,7 & - & - & 1 & 2,7 & 2 & 5,4 \\
\hline Enfermería Perinatal & 1 & 2,7 & - & - & 2 & 5,4 & 3 & 8,10 \\
\hline Total & 18 & 48,64 & 4 & 10,8 & 15 & 40,54 & 37 & 100,0 \\
\hline
\end{tabular}

Fuente: base de datos propia

En la tabla 1, prevalece la escogencia de temáticas de tipo gerencial en el área de Obstetricia $(f i=12$, $h i=32,43)$. El eje menos atractivo es el de educación $(f i=4, h i=10,8)$. Respecto del eje clínico se destaca que también la línea más atractiva es el de enfermería obstétrica $(f i=8, h i=21,62)$. Por último, es importante resaltar que la línea menos investigada corresponde a la enfermería ginecológica y andrológica $(f i=2, h i=5,4)$, seguida por la línea de Enfermería perinatal $(f i=3, h i=8,10)$.

\section{Enfoque cualitativo}

\section{Dimensión teórica}

Respecto del propósito y la pregunta de investigación, se observó que de 22 trabajos finales de graduación presentados bajo este enfoque, solo 11 establecían claramente ese rubro. Además, se encontró que nueve de los trabajos no definían en forma clara los objetivos.

En relación con la literatura, el marco teórico (referencial o conceptual), en 11 de los trabajos fue suficiente para el análisis de los resultados, en ocho no fue suficiente y en tres no se especificó adecuadamente este apartado.

Según la coherencia de la investigación con sus objetivos planteados y la pertinencia para sustentar la investigación, se encontró que de 22 investigaciones aplicadas, solo 12 mostraron coherencia y solo en siete, pertinencia. 


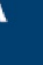

r

(

\section{Revista Electrónica Enfermeria Actual de costa Rica}

Otro aspecto valorado fue la bibliografía citada: en 11 trabajos, han transcurrido cinco años al periodo de la presentación del estudio, mientras que en otros 11 , la literatura cuenta con más de cinco años desde su publicación. En lo que respecta a la citación de los autores, se observó que 20 de los trabajos cumplían con este aspecto y tan solo dos de ellos no la presentaron.

Acerca del análisis de los resultados, interesó conocer si respondían a las unidades de análisis propuestas, si hubo reflexión crítica a partir de los datos encontrados y un discurso lo suficientemente profundo, acorde con un nivel de maestría. Se observó que siete trabajos no presentaban unidades de análisis, por ende, fue difícil emitir un criterio al respecto. Además, en 13 de ellos no se evidenció un discurso reflexivo crítico a partir de los resultados y en otros 13 tampoco se apreció profundización discursiva.

\section{Dimensión social}

En cuanto a esta dimensión, el objetivo radicó en conocer acerca de la publicación científica de los trabajos, la devolución de los resultados a los participantes de la investigación, así como si las recomendaciones sugeridas en la investigación fueron implementadas para facilitar el cambio en las intervenciones. También se valoró si se explicitaba las consideraciones éticas, los alcances y las limitaciones y si las conclusiones y recomendaciones presentaban en un formato adecuado.

Respecto de lo anterior, 13 de los trabajos no se han publicado y nueve de ellos no se conoce si han sido publicados; dichos resultados también se repiten en lo que respecta el medio de divulgación o revista científica. Además, 18 de estas investigaciones no evidencian en su texto haber realizado devolución a los participantes: tan solo cuatro lo mencionan.

En lo que se refiere a las recomendaciones sugeridas en la investigación, es importante acotar que en 16 de los trabajos no fueron implementadas: tan solo seis abordan dicho y aspecto y solo una investigación a la fecha de este estudio mantiene las recomendaciones.

Sobre las consideraciones éticas, 16 de los trabajos no aluden a este apartado, seis lo mencionan pero solo en términos de consentimiento informado, mientras que 14 de las investigaciones no explicitan los alcances y las limitaciones. Finalmente, se constató que 18 de los estudios guardan un formato adecuado para plantear las conclusiones y recomendaciones.

\section{Dimensión metodológica}

En este apartado, se destaca aspectos importantes en términos de diseño del estudio, método estructural cualitativo utilizado, si se explicitaba la aproximación cualitativa al objeto de estudio, así como los criterios de selección de los participantes, si se menciona y describe los instrumentos para recolectar información de acuerdo con el método cualitativo utilizado, las unidades y categorías de análisis, el tipo de triangulación y el procedimiento para el procesamiento de la información. 
Iniciando con el diseño utilizado, se observó que sólo 11 trabajos hacen mención del mismo y lo desarrollan. El método estructural cualitativo es explícito en ocho investigaciones; la aproximación epistemológica al objeto de estudio se mencionó en seis.

Por último, los criterios de selección de los participantes se evidencian en nueve trabajos, en 17 de los estudios se mencionó los instrumentos y técnicas para la recolección de información, así como en 15 se indica las unidades y categorías de análisis. Es relevante acotar que tan solo en dos investigaciones se menciona el tipo de triangulación utilizada para el análisis de los datos, mientras que el procesamiento de la información se describe en cinco de los trabajos.

\section{Enfoque cuantitativo}

\section{Dimensión teórica}

En esta dimensión se valoró si eran explícitos y claros el propósito y/o pregunta de investigación, al igual que los objetivos. En relación con el marco teórico (referencial o conceptual), se observó la suficiencia para el análisis de los resultados y la coherencia de acuerdo con los objetivos planteados. En cuanto a la bibliografía, se verificó si tenía más de cinco años, respecto de la presentación del trabajo y si se utilizó adecuadamente el sistema de referencias según los parámetros establecidos por la American Psychological Association (APA). En lo que respecta al análisis de los resultados, se corroboró si responden a los objetivos propuestos, si el análisis tiene suficiencia a partir de los datos presentados y si en el marco teórico hay evidencia de un discurso reflexivo crítico y el nivel de profundización necesario. Finalmente, se revisó si las tablas y gráficos fueron presentados en un formato correcto.

En lo que respecta al propósito o pregunta de investigación, en 12 de 15 investigaciones aplicadas (TFG), se establece de forma clara. En 13 TFG quedan claramente explícitas las preguntas de investigación.

Luego, en lo que toca al marco teórico (referencial o conceptual), solo en nueve de los 15 TFG se consideró que era suficiente para el análisis de los resultados; sin embargo, en 12 de ellos se encontró coherencia temática según los objetivos planteados en la investigación. Por otro lado, según la pertinencia, en 10 TFG está presente dicha variable.

Por otra parte, de la bibliografía citada en la investigación, siete TFG tiene cinco años de existencia a la defensa pública de la investigación; en cuanto a como fue citada, en 13 trabajos fue acorde con los parámetros establecidos según el sistema de referencia usado.

Otra variable fue el análisis de resultados: de 15 TFG analizados, solo ocho responden a los objetivos planteados y siete evidencian análisis a partir de los datos encontrados. El discurso reflexivo crítico se detectó tan solo en seis de los trabajos, lo mismo para el nivel de profundización necesario en una maestría.

Finalmente, se revisaron las tablas y los gráficos: siete de los 15 TFG se presentaron en un formato correcto de acuerdo con las recomendaciones de estadística. 


\section{Dimensión social}

En este apartado fue interesante conocer aspectos relacionados con la publicación científica del trabajo, la implementación de recomendaciones sugeridas por la investigación, así como valorar el impacto social, la presencia explícita de consideraciones éticas, los alcances y limitaciones y si las conclusiones fueron redactadas adecuadamente.

En cuanto a la publicación científica, ningún TFG revisado fue publicado.

Respecto de los criterios éticos en la investigación, dentro de los 15 textos analizados no se evidencia la devolución de resultados a los sujetos participantes en el estudio, aparte de que solo en cuatro TFG, se indica las consideraciones éticas.

Otro aspecto importante de análisis fue las conclusiones y recomendaciones. En 12 TFG están explícitos ambos elementos, mas en el resto (3) se anotan consideraciones finales solamente. Respecto del formato y redacción, nueve TFG presentaron una forma adecuada. Es destacable que en ningún estudio se aclara si las recomendaciones sugeridas en la investigación fueron acatadas o si tuvieron algún impacto social.

Finalmente, solo en cinco TFG se observa los alcances y limitaciones.

\section{Dimensión metodológica}

En esta dimensión se consideró las siguientes variables: diseño y tipo de investigación, la congruencia con el problema planteado, el planteamiento de la población y el tipo de muestreo, la anotación de criterios de inclusión y exclusión, la presencia y redacción adecuada del sistema de variables de acuerdo con los objetivos propuestos, presencia de hipótesis, si hay evidencia del desarrollo de instrumentos, técnicas para la recolección de información, el formato de los instrumentos, el tipo de validación instrumental y, finalmente, el detalle del procesamiento de los datos.

En cuanto al diseño utilizado, tan solo en 10 TFG se menciona el tipo de investigación, mas en 13 TFG sí queda explícito y es congruente con el problema y los objetivos planteados.

Se evidencia la población y la muestra en 13 de las investigaciones, mas solamente en ocho de ellas se presentan criterios de inclusión y exclusión de participantes.

En lo que respecta al sistema de variables, aparece en 11 TFG; sin embargo, solo 10 detallan la definición conceptual, operacional e instrumental. En ocho trabajos se señala los indicadores y la definición operacional de las variables en términos adecuados de medición. Acerca de este tema, se recalca que el sistema de variables muestra coherencia con los objetivos del estudio solo en 10 TFG.

Solamente en dos investigaciones se anota hipótesis.

Respecto de los instrumentos y técnicas para recolectar información, en 13 de las investigaciones se anota con detalle; sin embargo, la elaboración de los instrumentos evidencia que solo ocho presentan el formato 
adecuado según los parámetros que brinda la estadística. El tipo de validación instrumental es mencionado en siete trabajos .

Finalmente, en 10 TFG se detalla el procedimiento (manual o digital) para el procesamiento de los datos.

\section{DISCUSIÓN}

Para las maestrías profesionales y como requisito de graduación, el Sistema de Estudios de Posgrado (SEP) de la Universidad de Costa Rica establece la investigación aplicada según reza en el documento "Lineamientos de trabajos finales de investigación aplicada", acerca de las que las comisiones de cada programa de posgrado se encargan de establecer las características concretas de investigación que el estudiantado debe cumplir (Universidad de Costa Rica, SEP, 2007).

La maestría en Enfermería Ginecológica, Obstétrica y Perinatal (GOP) fue creada en el año 2000: la primera promoción se graduó en el año 2002. Hasta este año, se han realizado 12 promociones con grupos numerosos de estudiantes de diferentes áreas del país. Sin embargo, en términos de los alcances de la investigación realizada a partir de los trabajos de investigación, los resultados son poco alentadores.

En la descripción de sus ejes y líneas de investigación, La maestría GOP menciona que

ha establecido cuatro líneas orientadoras para la investigación en las áreas que competen a la maestría. Al trabajar en una línea de investigación, las y los interesados se comprometen a desarrollar tópicos convergentes y complementarios al eje investigativo. Además de tener plena conciencia de la necesidad inherente que trae el investigar como lo es la publicación científica. Debido a que mediante la publicación se puede evidenciar el trabajo realizado, divulgar resultados, generar cambios, someterse a la crítica constructiva de la comunidad científica y profesional. La publicación científica se convierte en el indicador más confiable de que el trabajo que se está realizando en una línea de investigación conduce al logro de metas y objetivos claros y resultados significativos (Bonilla, L., s.f., s.p.).

Dichos ejes son educativo, clínico y gerencia y en cada uno se estableció líneas de investigación que responden a la Salud Sexual y Salud Reproductiva, Derechos Sexuales y Derechos Reproductivos, Enfermería Ginecológica y Andrológica, Enfermería Obstétrica y la Enfermería Perinatal.

A partir de la investigación educativa se genera una serie de retos que dan respuesta a las necesidades que existen en la enseñanza de la enfermería en general y, en el caso particular de esta investigación, en la Enfermería Ginecológica, Obstétrica y Perinatal, así como también en la práctica clínica, el abordaje teórico y todos los componentes que forman un plan de estudios aunque, en especial, la valoración dicotómica entre la teoría y la práctica.

Por ser una maestría profesional (trata de resolver problemas de su práctica) su particularidad disciplinar subyace en la "utilidad aplicable del conocimiento que produce", por tanto, impera "una necesaria subordinación del conocer con respecto al hacer" (Villegas, 2011, p.92). 
Como la identifica su nombre, desde lo teórico, la maestría enfatiza aspectos ginecológicos, obstétricos y perinatales; a pesar de ello, en el desarrollo de los diferentes cursos que se imparten sobresale el abordaje obstétrico, un condicionante que se ve reflejado en las preferencias del estudiantado por llevar a cabo investigaciones en la línea de investigación obstétrica.

Aun así, es necesario un equilibrio entre las diferentes áreas de la maestría, dado que en las tres áreas existen necesidades e interrogantes interesantes que merecen ser investigadas, más si se considera que la investigación mejora la práctica y esta influye en los diferentes campos del saber para plantear nuevas investigaciones, refutar conceptos, entre otros aspectos. Al respecto, Vargas (2009) expresa que,

Situar a la persona en un contexto, visto como el medio al que pertenece, integrado por la cultura y en la cultura la humaniza, observa, percibe, aprende y actúa; por lo tanto, es el que crea una realidad propia que puede ser intervenida, mejorada o transformada por quien investiga, emplea la práctica como investigación y ésta, a su vez, como práctica (p.158).

Otro factor que puede influir en la escogencia por investigar en un eje y una línea de investigación determinada se refiere al docente, quien es mediador o productor del conocimiento y que redirecciona las decisiones del estudiantado para abordar las diferentes necesidades o temas de investigación; no cabe duda, que existen también intereses predominantes de parte del profesor (a) en términos de temas y metodología investigativa.

El aspecto mencionado podría ser un determinante en cuanto a que el estudiante no concluya su trabajo final de graduación, al responder a las preferencias del tutor y no a su propia necesidad investigativa. En cuanto al trabajo final de graduación, según Escalante (2009), "representa la puesta en escena de procesos de construcción intelectual y aprendizaje avanzados, según las orientaciones que genere cada universidad, el cual se asocia a lo que significa trabajar intelectualmente en un proceso avanzado de aprendizaje y de cultura académica" (p.10).

Aparte de todos los beneficios que se obtiene de la investigación, hay que rescatar que "el impacto social y económico de la investigación es un referente básico en las directrices de las políticas, considerándose también un aspecto fundamental en la evaluación científica" (Colás, 1997, p.1).

En el caso de la investigación en la maestría -además de productora de conocimiento-se le considera una forma de práctica creativa, dado que enfermería es una profesión eminentemente práctica en la que los trabajos de graduación que surgen de un ambiente académico con ciertas restricciones para investigar en los diferentes campos clínicos, se convierten en réplicas para graduarse sin un verdadero impacto social que inste al cambio de la realidad que se investiga. Por tal motivo, algunos temas que se leen y aparentan atractivos e interesantes quedan para ocupar un espacio en la biblioteca de la universidad, a lo que hay que agregar la escasa o nula divulgación y publicación científica de los trabajos investigativos.

El análisis de este estudio develar que, a pesar de los esfuerzos de la maestría para equiparar en importancia las áreas temáticas - ginecología, obstetricia y perinatología- en la rotación clínica -en término de tiempo- el énfasis que existe se refiere al quehacer propiamente obstétrico, por lo cual no es de extrañarse que haya una fuerte inclinación por pensar que el especialista en enfermería egresado de la maestría empieza en 
obstetricia y termina en obstetricia; incluso, cuando se les pregunta respecto de su especialidad, el profesional responde "soy enfermera (o) obstetra", lo cual marca la percepción identitaria de los egresados e influye en sus intereses investigativos.

Dentro del mismo eje de enfermería obstétrica, se detecta una predilección por el eje de gerencia (12 TFG), seguido por el eje clínico ( $8 \mathrm{TFG);} \mathrm{sin} \mathrm{embargo,} \mathrm{tal} \mathrm{dato} \mathrm{está} \mathrm{en} \mathrm{entredicho} \mathrm{porque,} \mathrm{como} \mathrm{se} \mathrm{mencionó,} \mathrm{los}$ temas de investigación -en muchas ocasiones y en años anteriores- son propuestos por el tutor (cuyo interés aparenta centrarse en el eje gerencial), oportunidad en la que se graduaban de seis a nueve estudiantes, incluso. Esta propuesta era muy atractiva para el estudiantado porque el esfuerzo era mínimo, dado que compartiría un tema elegido por el tutor con un grupo considerablemente grande en el que se trabajaría, cuando mucho, en un solo objetivo.

A la situación descrita hay que añadir que con tales trabajos finales de graduación tampoco se ha mejorado la práctica profesional en términos de mejorar la calidad de atención a las personas: su relevancia radica en que han servido para que se gradúe un numeroso grupo de estudiantes.

De lo mencionado nace la inquietud de este estudio respecto del verdadero desarrollo de la investigación y las implicaciones para la práctica en la maestría a partir de los trabajos finales de graduación.

Este estudio analizó los dos enfoques predominantes en los que se desarrolla los trabajos finales de graduación y enfatiza tres dimensiones: la teórica, la social y la metodológica.

En cuanto a los trabajos finales de graduación basados en el enfoque cualitativo, es importante acotar que desde el S. XIX hasta la actualidad han surgido distintas formas de investigar partiendo del paradigma naturalista, desde el que -el investigador (a) trata de recolectar información desde el sitio natural en donde ocurre el suceso objeto de estudio, idea que rescata Le Compte (1995), citado por Rodríguez, Gil y García (1996), quien considera

los términos utilizados para conceptualizar cada uno de estos enfoques denotan la importancia de los constructos participantes, o los significados que los sujetos de la investigación asignan a sus acciones, el contexto del estudio, la relación entre el investigador y los que están siendo estudiados, los procedimientos y técnicas para la recogida de datos, los tipos de evidencias aducidas en apoyo de las afirmaciones realizadas, así como los métodos y la importancia del análisis utilizado (p.1).

Considerando lo mencionado, se rescata la idea de que quien investiga desde este paradigma requiere dominio teórico y práctico que confiera a los estudios validez y el suficiente peso teórico, metodológico y social para que exista un verdadero impacto y cambio en la realidad que se investiga. De modo contrario, las investigaciones serían superfluas, carentes de impacto y con serios problemas metodológicos, razón por la que, según Denzin y Lincoln (1994) el investigador cualitativo se somete a "una doble tensión simultánea :por una parte, es atraído por una amplia sensibilidad, interpretativa, postmoderna, feminista y crítica; por otra, puede serlo por unas concepciones más positivistas, postpositivistas, humanistas y naturalistas de la experiencia humana y su análisis" (p.576) . 
Entonces, todo investigador cualitativo debe tener un amplio dominio tanto para establecer su pregunta o preguntas de investigación, realizar la búsqueda de literatura respectiva que sustente el estado de la cuestión y plantear sus objetivos los cuales lo guiarán a través de todo el proceso. Es lamentable que tan solo un 50\% de las investigaciones aplicadas y analizadas presentaban una adecuada pregunta de investigación y un planteamiento coherente y pertinente con los objetivos planteados, así como que en un $50 \%$ el referente bibliográfico era insuficiente .

Otro aspecto que es importante resaltar es la poca profundización en el análisis de datos que se evidenciaban en siete de los trabajos finales; es decir, no hubo dominio del discurso reflexivo cualitativo. De igual forma, fue sorprendente determinar la ausencia de unidades de análisis, aspecto obligatorio para una estructura y ordenamiento del proceso de análisis y reflexión de los datos pesquisados. Al respecto, cabe indicar que el dato cualitativo representa información de la realidad que se investiga y que debe ser expresado mediante un profundo proceso de comunicación creativa en forma de texto. Este análisis de la información permite al investigador (a) discriminar, establecer relaciones, generar interpretaciones o modelos sobre la realidad estudiada (Rebolledo, s.f.).

En cuanto a la dimensión social, los resultados confirman la escasa o nula publicación científica, hecho señalado por Calvo (2006), quien agrega que

la divulgación de la ciencia constituye virtualmente un sistema de conocimiento, cuyo principio rector es la reformulación clara, amena y delimitada del conocimiento científico, de sus resultados y de su método (Alboukrek, 1991) y a la vez una forma especial de transmitir este conocimiento (Beltrán, 1983). Si el científico es responsable del rigor, el mediador entre la ciencia y la sociedad es uno de los elementos indispensables de esta dimensión básica de nuestro tiempo que es la difusión del conocimiento (p.1).

En otros ámbitos o países, la divulgación forma parte implícita del quehacer científico, sin embargo, en la Enfermería en Costa Rica no sucede así, debido a que es difícil que el estudiantado divulgue los resultados de su trabajo final de graduación; incluso, los docentes consideran un acto casi mesiánico que el estudiante concluya su tesis y redacte un artículo científico para que sea sometido al respectivo arbitraje en una revista científica.

Un factor que puede estar influyendo en la falta de divulgación científica y publicación es que el Sistema de Estudios de Posgrado de la Universidad de Costa Rica no tiene una normativa al respecto, por tanto, ni siquiera por requisito académico se fomenta la elaboración del artículo científico, lo cual redunda en una invisibilización del quehacer investigativo en las maestrías que, a su vez, genera un pobre impacto social.

Por otro lado, en relación con las recomendaciones anotadas en el documento, no hay suficiente evidencia o descripción que insinúe que serán tomadas en cuenta por los interesados y ejecutores del mejoramiento en la práctica reflejado por la investigación científica; por consiguiente, se genera un vacío en cuanto a la información que se pueda analizar desde el documento elaborado. 
.

Un aspecto interesante que las investigadoras de este estudio encontraron fue la carencia de un apartado en el que se mencione las consideraciones éticas de la investigación, en este caso, tan solo se observó que seis de los trabajos aplicaron el consentimiento informado.

Con respecto a la elaboración de trabajos finales de graduación que han utilizado la metodología cuantitativa, se colige que es la menos escogida por los estudiantes, lo cual puede ser provocado por la misma influencia de las preferencias del tutor asignado.

Este diseño metodológico es propio del paradigma positivista que se propone verificar y contrastar datos y buscar causas, por lo tanto, requiere de precisión secuencial, conocimiento estadístico y de una hipótesis claramente establecida, lo cual exige que el investigador que escoge esta metodología la desarrolle con gran rigurosidad. Al respecto, Hernández y otros (2010) afirman que "desde el enfoque cuantitativo, y si hemos seguido paso a paso el proceso de investigación, es natural que las hipótesis surjan del planteamiento del problema, es decir, provienen de la revisión de la literatura" (p.93). Considerando lo anterior se observó que la mayoría de investigaciones cuantitativas elaboradas en la maestría GOP, carecen de claridad metodológica y no formulan hipótesis, por tal razón es imposible tener claridad del alcance de la investigación y, por consiguiente, es muy posible que haya errores en los resultados, cuya consecuencia es que se desarrolla investigaciones sin valor científico.

Entonces, no es de extrañarse que ante la falta de precisión metodológica, se encuentre memorias que no desarrollan adecuadamente las variables de investigación, debido a que no concuerdan con los objetivos planteados o presentan grandes ambigüedades en el desarrollo de los instrumentos de recolección de datos.

Estos hallazgos en la dimensión metodológica son relevantes, pues evidencian la necesidad de fortalecer las competencias de investigación, de tal forma que se cumpla con la responsabilidad de mejorar la práctica de la enfermería GOP en Costa Rica, a través del conocimiento actualizado, máxime que, según Triviño y Sanhueza (2005), "Enfermería tiene el desafío de generar sus propios conocimientos a través de la investigación y construir su realidad desde su propia perspectiva, es decir, su práctica y objeto disciplinar, que es el cuidado" (p.22).

En cuanto a la dimensión social investigada, las investigaciones de enfoque cuantitativo no fueron publicadas: es posible suponer que se desarrollaron solamente con el objetivo de cumplir con un requisito de graduación, lo cual es preocupante pues el fin propio de la investigación no está siendo considerado ni fomentado por los tutores, lo cual es enfatizado por Vásquez (2011), al comentar lo siguiente:

Ahora bien, sabemos que para la disciplina de enfermería es indispensable generar conocimiento para crear y mantener una sólida base científica para la práctica, que promueva mejores resultados en los cuidados de las personas sujeto de cuidado, y que refleje, no solo el ejercicio de una práctica fundamentada científicamente, sino además el desarrollo de una profesión autónoma. Por ello, el deber y sentido de continuar avanzando en la búsqueda y generación de conocimiento de cuidar como esencia ontológica de la enfermería y, en ese sentido, explicar y demostrar la realidad del fenómeno, así como buscar relaciones, generalizar y validar lo relativo al cuidado, es una tarea permanente y comprometida que las enfermeras debemos tener por el sentido mismo que esta labor entraña (p.177). 
Por consiguiente, la ausencia de divulgación del estudio de un evento implica la imposibilidad de validar su impacto; entonces, es de esperar que, tal y como se evidencia en los informes escritos, no aparezcan desarrollados los alcances y limitaciones. Se podría pensar que los estudiantes cumplen una exigencia curricular solamente y no interiorizan los requisitos éticos del investigador, aparte de que las poblaciones e instituciones participantes no reciben devolución de su intervención: dichos vacíos son esperables y casi predictivos por la ausencia de apartado de las consideraciones éticas en los informes finales de las investigaciones. Para la Enfermería, es imprescindible solventar dicha carencia, especialmente en un informe que es requisito final de un programa de maestría, respecto de lo que Emanuel (1999) afirma lo siguiente:

No solo deben los investigadores clínicos estar capacitados en las metodologías apropiadas, también deben estar capacitados en lo que se refiere a la adherencia a estos requisitos éticos. De igual manera, debido a que la evaluación independiente de la investigación clínica debe su valor, validez, criterios de selección, proporción de riesgo-beneficio, documentos de consentimiento informado y procedimientos para monitorear a los sujetos inscritos, las aptitudes necesarias deben variar del conocimiento científico al ético (p.92).

Respecto a la dimensión teórica analizada en las investigaciones de corte cuantitativo, se evidenció un insuficiente análisis de los datos en cuanto a profundidad. Un aspecto que provocaría una profundidad analítica insuficiente puede ser la inadecuada presentación de los datos, evidente en la mitad de los informes, a lo que hay que agregar que el formato incorrecto de tablas y gráficos imposibilita visualizar la información.

Respecto de la investigación cuantitativa, el análisis de los datos se define como "cualquier tratamiento matemático de los datos finales que permita trazar comparaciones o relaciones numéricas. El término abarca, por consiguiente, tanto análisis simples, descriptivos, como análisis propios de estadística inferencial" (Arellano, 1990, p.151). Dado lo anterior, no es raro que en los informes finales de graduación, de los datos se colijan conclusiones válidas, replicables en un contexto determinado. Sin embargo, el discurso reflexivo desarrollado en las investigaciones aplicadas analizadas, no permite esta generalización del conocimiento. Este vacío puede ser resultado de la influencia del uso de bibliografía poco renovada y marcos teóricos insuficientes.

En síntesis, el panorama respecto del tema de "investigación” en la maestría GOP lejos de aportar conocimiento en enfermería y desarrollo disciplinar, se limita a ser un requisito de graduación, un limitado espacio para generar conocimiento poco innovador que no contribuye al mejoramiento de las prácticas GOP a partir de la investigación, situación que motiva el desarrollo de estrategias que contribuyan al fortalecimiento académico, una mayor participación docente, de investigadores y estudiantes en los que la investigación sea considerada una función sustantiva que impulse el avance científico y genere buenas prácticas de salud sexual y salud reproductiva en el país.

\section{CONCLUSIÓN}

De la investigación realizada se colige las siguientes conclusiones:

- En la investigación en la Maestría en Enfermería Ginecológica, Obstétrica y Perinatal, específicamente en los trabajos de graduación que surgen de un ambiente académico con ciertas 
restricciones para investigar en los diferentes campos clínicos, los estudios se constituyen en réplicas de otros cuyo único objetivo es cumplir con el requisito de graduación, mas sin que haya un verdadero impacto social que inste al cambio de la realidad que se investiga.

- Con respecto a la elaboración de trabajos finales de graduación que utilizan la metodología cuantitativa, se constata que es la modalidad menos escogida por los estudiantes.

- Se evidencia falta de precisión metodológica en la mayoría de los trabajos.

- Existe una gran falta de interés por la publicación de los trabajos de investigación, lo cual redunda en la poca proyección social de los resultados.

- Se evidenció falta de profundización tanto en los marcos referenciales como en el análisis de los datos.

- La investigación en la maestría GOP no aporta conocimiento enfermero y desarrollo disciplinar, sino que se muestra solo como requisito de graduación, por lo que no contribuye al mejoramiento de las prácticas GOP a partir de la investigación.

\section{Agradecimiento}

Nuestro más sincero agradecimiento al personal administrativo del Posgrado en Ciencias de la Enfermería por su ayuda en cuanto a recolectar los trabajos de investigación y su anuencia a colaborar en otros asuntos administrativos necesarios para elaborar esta investigación.

\section{REFERENCIAS BIBLIOGRÁFICAS}

American Association of Colleges of Nursing (1996). The essential of master's education for advance practice nursing. Washington, D.C: AACN.

Arellano J. (1990). Elementos de la Investigación a través de su informe. San Jose: EUNED.

Barnsteiner, J. (2011). Workplace abuse in nursing: Policy strategies. In D. Mason, J. Leavitt, M. Chafee (Eds.), Policy \& politics in nursing and health care (6th Ed.). (428-434). St. Louis, MO: Elsevier Saunders.

Bonilla, L. (s.f.) La línea de investigación. Papel de trabajo para el investigador novel. Recuperado de: www.monografías.com/trabajos17/linea-de-investigación/linea-de-investigacion.html

Calvo, M. (2006). Objetivos de la Divulgación Cientifica. Recuperado de http://www.manuelcalvohernando.es/articulo.php?id=66

Colás, P. (1997). La investigacion en la práctica. Revista de Investigacion educativa, 15 (2) 119-142.

CONARE (2001). Dictamen sobre la propuesta de creación de la maestría en Enfermería Ginecológica, Obstétrica y Perinatal de la Universidad de Costa Rica. Universidad de Costa Rica. 
Consejo Nacional de Rectores Oficina de Planificación de la Educación Superior (2001) Dictamen sobre la propuesta de creación de la maestría en Enfermería Ginecológica, Obstétrica y Perinatal en la Universidad de Costa Rica. OPES-23/2001.

Denzin, N. y Lincoln, Y. (1994) Handbook of qualitative research. Londres: Sage

Emanuel, E. (1999). ¿Qué hace que la investigación clínica sea ética? Siete requisitos éticos. Pellegrino Filho A, Macklin R. Investigación en sujetos humanos: experiencia internacional. Santiago de Chile: Programa Regional de Bioética OPS/OMS, 39.

Escalante, E. (2009) Aproximación cualitativa a las experiencias de los alumnos de posgrado. Revista Académica 37, 8-31.

Fain, J. (2004). Reading, Understanding, and Applying Nursing research. Massachusetts: F. A. Davis Company.

Fawcett, J., Garity, J. (2009). Evaluating research for evidence-based nursing practice. Phyladelphia: F.A. Davis Company.

Maestría Ginecológica, Obstétrica y Perinatal (2013). Folleto de la Maestría Ginecológica, Obstétrica y Perinatal Sistema de Estudios de Posgrado. Universidad de Costa Rica.

Hernández, R., Fernández, C., Baptista, P. (2010). Metodología de la investigación. México D.F.: Editorial Mc Graw Hill.

Rebolledo, C. (s. f.) El Análisis en investigación cualitativa. Organización y Reducción de la información. Facultad de Educación y Humanidades. Universidad del Bío Bío. Chile

Rodríguez, G., Gil, J., García, E. (1996) Metodología de la investigación cualitativa. Granada: Ediciones Aljibe.

Truisi, M. (2011). Cuidar e investigar: desafíos metodológicos en enfermería. Texto and Contexto Enfermagem, $20(1), 175$.

Triviño, Z., Sanhueza, O. (2005). Paradigmas de investigación en enfermería. Ciencia y enfermería, 11(1), 17-24.

Vargas, Z. (2009) La investigación aplicada: una forma de conocer las realidades con evidencia científica. Revista Educación 33 (1), 155-165.

Vásquez, M. (2011). Cuidar e Investigar: Desafíos metodológicos en Enfermería. Texto Contexto Enfermagen, Jan-Mar, 20(1), 175-183.

Villegas, C. (2011). La maestría en Trabajo social y la investigación: Análisis de la producción de conocimiento a partir de los trabajos finales de graduación. Reflexiones 90 (2), 89-100 